$18,12,19$

\title{
Влияние взаимодействия слоев на жесткость изгибных деформаций многослойных углеродных нанолент
}

\author{
(C) А.В. Савин ${ }^{1,2}$, О.И. Савина ${ }^{2}$ \\ ${ }^{1}$ Институт химической фризики им. Н.Н. Семёнова РАН, \\ Москва, Россия \\ ${ }^{2}$ Российский экономический университет им. Г.В. Плеханова, \\ Москва, Россия \\ E-mail: asavin@center.chph.ras.ru \\ (Поступила в Редакцию 27 ноября 2018 г. \\ В окончательной редакции 28 ноября 2018 г. \\ Принята к публикации 28 ноября 2018 г.)
}

\begin{abstract}
Исследовано влияние слабого невалентного взаимодействия слоев на устойчивость к изгибу многослойной наноленты графена. Численное моделирование изгиба конечной многослойной наноленты и анализ ее изгибных колебаний показывают, что взаимодействие слоев приводит к заметному повышению нормированной на число слоев изгибной жесткости только для нанолент длины $L>12 \mathrm{~nm}$. Чем больше длина, тем сильнее происходит это повышение. Так при длине $L=24 \mathrm{~nm}$ взаимодействие слоев приводит к увеличению изгибной жесткости в три раза для двухслойной и в шесть раз для девятислойной наноленты. Поэтому использование многослойных нанолент может позволить существенно увеличить устойчивость к изгибу протяженных наноконструкций.
\end{abstract}

Исследование выполнено при финансовой поддержке Российского научного фонда (проект 18-53-05022). Вычислительные ресурсы предоставлены межведомственным суперкомпьютерным центром РАН.

DOI: 10.21883/FTT.2019.04.47433.329

\section{1. Введение}

Атомы углерода способны создавать колоссальное разнообразие структур, среди которых в последнее время большое внимание исследователей привлекает моноатомный кристаллический слой - графен [1-7]. Данный наноматериал демонстрирует высокую электро и теплопроводность, рекордные жесткость и прочность на разрыв, колоссальную удельную поверхность и другие свойства, обеспечивающие большое разнообразие его потенциальных применений. Механические свойства графена могут быть использованы для построения различных датчиков. Слабая устойчивость к изгибу листа графена может быть использована для построения высокочувствительных датчиков давления [8-11].

Важным свойством однослойного листа графена является его низкая изгибная жесткость, допускающая возможность создания складок и рулонов, устойчивость которых обеспечивают относительно слабые ван-дерваальсовы взаимодействия атомов углерода в $s p^{2}$-структурах. В многослойном листе графена ван-дер-ваальсовы взаимодействия атомов соседних слоев повышают устойчивость плоской формы листа. Естественно ожидать, что жесткость изгибной деформации $K$-слойного листа должна быть в $K$ раз больше жесткости однослойного листа (жесткости одного слоя). В данной работе будет показано, что это справедливо только для нанолент графена длины $L<12 \mathrm{~nm}$. При таких размерах жест- кость изгиба многослойной наноленты, нормированная на ее толщину, практически не зависит от числа слоев. При больших длинах взаимодействие слоев приводит к существенному увеличению нормированной жесткости изгиба наноленты. Так при $L=24 \mathrm{~nm}$ взаимодействие слоев приводит к увеличению жесткости в 3 раза для двухслойной и в 6 раз для девятислойной наноленты.

Для моделирования динамики листов графена обычно используют полноатомные модели. Такие модели требуют значительных компьютерных ресурсов и не позволяют рассматривать динамику длинных нанолент на значительных отрезках времени. Сложность полноатомных моделей также затрудняет проведение полного анализа возможных стационарных упаковок длинных нанолент. Для преодоления этих трудностей была предложена цепная модель наноленты, учитывающая ее продольную и изгибную деформацию, а также ван-дер-ваальсовы взаимодействия ее атомов [12,13]. В рамках этой модели были описаны возможные стационарные состояния рулонных упаковок графеновых нанолент, исследованы их энергетические характеристики, рассчитаны низкие частоты колебаний. В настоящей работе данная модель будет использована для оценки влияния взаимодействия слоев на жесткость изгибных деформаций многослойных нанолент. Жесткость изгибных деформаций будет оценена через нахождение частот собственных изгибных колебаний наноленты, так и путем прямого нахождения зависимости энергии наноленты от величины ее изгиба. 


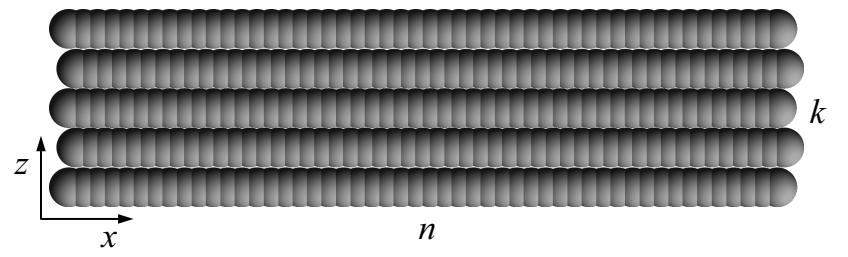

Рис. 1. Цепная модель пятислойной наноленты графена. Число слоев $K=5$, число звеньев в каждой цепи $N=50$, индекс $k$ нумерует цепочку, $n-$ узел в цепочке.

\section{2. Цепная модель многослойной наноленты}

Графеновая нанолента представляет собой ленту постоянной ширины, вырезанную из плоского листа графена. Для упруго изотропного графена продольная и изгибная жесткости наноленты слабо зависят от ее ориентации. Для определенности рассмотрим наноленту ориентации зигзаг ([13], рис. 1). Модель цепи, описывающая продольные и изгибные движения однослойной наноленты, подробно представлена в работах $[12,13]$. В работе [14] модель была использована для описания динамики многослойной наноленты.

Рассмотрим $K$-слойную наноленту расположенную вдоль оси $x$ параллельно плоскости $x y-$ см. рис. 1 . В цепной модели наноленты предполагается синхронное движение всех атомов, расположенных на одной поперечной линии (линии, параллельной оси $y$ ). В модели $K$-слойной наноленте соответствует система из $K$ цепей. Гамильтониан системы $K$ параллельных цепей (рис. 1 ) имеет форму

$$
\begin{aligned}
H & =\sum_{k=1}^{K}\left[\sum_{n=1}^{N} \frac{1}{2} M\left(\dot{x}_{n, k}^{2}+\dot{z}_{n, k}^{2}\right)+\sum_{n=1}^{N-1} V\left(r_{n, k}\right)+\sum_{n=2}^{N-1} U\left(\theta_{n, k}\right)\right] \\
& +\sum_{k=1}^{K-1} \sum_{l=k+1}^{K} \sum_{n=1}^{N} \sum_{m=n-N_{0}}^{n+N_{0}} W\left(r_{n, k ; m, l}\right)
\end{aligned}
$$

где $K$ - число цепей, $N$ - число звеньев в каждой цепи, $M=12 m_{p}-$ масса атома углерода $\left(m_{p}=1.6603 \cdot 10^{-27} \mathrm{~kg}-\right.$ масса протона), $\mathbf{u}_{n, k}=\left(x_{n, k}, z_{n, k}\right)-$ вектор, задающий положение $n$-го узла $k$-той цепи, $r_{n, k}=\left|\mathbf{v}_{n, k}\right|-$ расстояние между соседними узлами $n$ и $n+1$ цепи $k$ (вектор $\left.\mathbf{v}_{n, k}=\mathbf{u}_{n+1, k}-\mathbf{u}_{n, k}\right), \theta_{n, k}-$ угол между соседними звеньями цепи (угол меду векторами $\mathbf{v}_{n, k}$ и $-\mathbf{v}_{n-1, k}$ ), $r_{n, k ; m, l}=\left|\mathbf{u}_{n, k}-\mathbf{u}_{m, l}\right|-$ расстояние между узлами $(n, k)$ и $(m, l), N_{0}=20$ - число взаимодействующих звеньев соседних цепей. В последнем слагаемом сумма $n+N_{0}=N$, если $n>N-N_{0}$, и $n-N_{0}=1$, если $n \leq N_{0}$.

Первый член суммы (1) описывает энергию отдельных нанолент, второй - энергию межцепных взаимодействий. Продольная жесткость цепи задается гармониче- ским потенциалом

$$
V(r)=\frac{1}{2} K_{0}(r-a)^{2}
$$

где $a=1.228 \AA-$ шаг цепи (продольный шаг наноленты), $K_{0}=405 \mathrm{~N} / \mathrm{m}$ - жесткость межузельного взаимодействия. Изгибная жесткость цепи описывается потенциалом

$$
U(\theta)=\epsilon_{0}[\cos (\theta)+1]
$$

где косинус $n$-го „валентного“ угла $\cos \left(\theta_{n}\right)=$ $=-\left(\mathbf{v}_{n-1}, \mathbf{v}_{n}\right) / r_{n-1} r_{n}$, энергия $\epsilon_{0}=3.5 \mathrm{eV}$.

При этих значениях $K_{0}$ и $\epsilon_{0}$ дисперсионные кривые отдельной цепи наиболее точно совпадают с дисперсионными кривыми плоской наноленты графена, соответствующими ее продольным и изгибным колебаниям [12,13].

Потенциал $W\left(r_{n, m ; k, l}\right)$ описывает слабые невалентные взаимодействия узлов $(n, k)$ и $(m, l)$. Энергию взаимодействия узлов можно найти численно как сумму невалентных взаимодействий атомов углерода из соответствующих поперечных линий нанолент. Так как соседние поперечные линии отличаются положением на них атомов, вычисление энергии с использованием потенциала взаимодействия Леннарда-Джонса дают слегка отличающиеся потенциалы для узлов с четной и нечетной разницей индексов $n-m$. Их среднее значение для лент большой ширины с высокой точностью аппроксимируется потенциалом Леннарда-Джонса $(5,11)$

$$
W(r)=\epsilon_{1}\left[5\left(r_{1} / r\right)^{11}-11\left(r_{1} / r\right)^{5}\right] / 6
$$

с равновесной длиной связи $r_{1}=3.61 \AA$, энергией взаимодействия $\epsilon_{1}=0.0166 \mathrm{eV}$ (см. рис. 2).

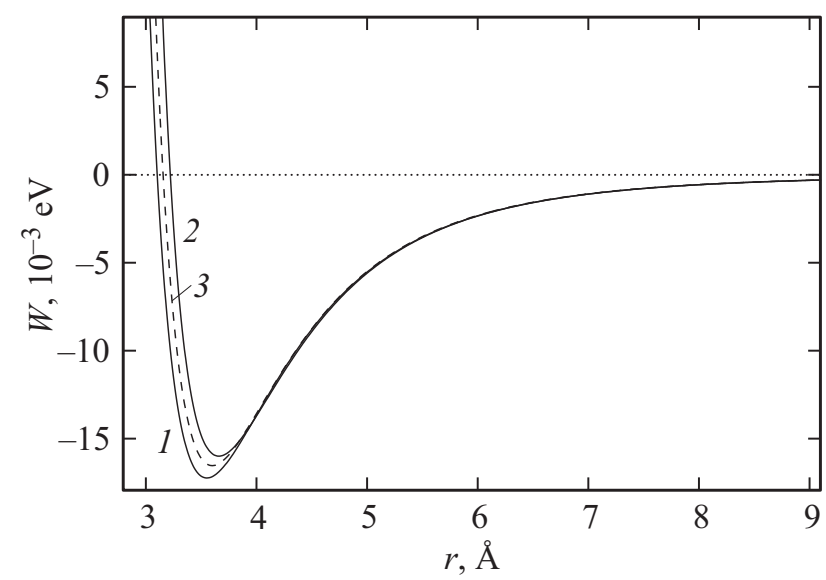

Рис. 2. Зависимость энергии взаимодействия поперечных линий атомов наноленты $W$ от расстояния между ними $r$ для линий, расположенных через нечетное и четное число продольных шагов наноленты (кривые 1 и 2). Пунктирная кривая 3 дает зависимость, описываемую потенциалом Леннарда-Джонса (4). 


\section{3. Дисперсионные кривые К-слойной цепи}

Рассмотрим систему $K$ параллельных циклических цепей из $N$ звеньев каждая - см. рис. 1. Для построения дисперсионных кривых данной молекулярной системы сначала найдем ее основное состояние, т. е. элементарную (поперечную) ячейку системы $\left\{\mathbf{u}_{0, k}\right\}_{k=1}^{K}$ и ее шаг $a_{0}$ (двумерный вектор $\mathbf{u}_{0, k}=\left(x_{0, k}, z_{0, k}\right)$ задает положение в ячейке атома $k$-той цепи). В основном состоянии атомы системы цепей будут иметь координаты

$$
\begin{gathered}
x_{n, k}^{0}=x_{0, k}+n a_{0}, \quad y_{n, k}^{0}=y_{0, k}, \\
n=1,2, \ldots, N, \quad k=1, \ldots, K .
\end{gathered}
$$

Координаты основного состояния будем искать численно как решение задачи на минимум потенциальной энергии основного состояния

$$
E \rightarrow \min : \quad\left\{\mathbf{u}_{0, k}\right\}_{k=1}^{K}, \quad a_{0} .
$$

Пусть $\left\{\mathbf{u}_{n, k}^{0}\right\}_{n=1, k=1}^{N, K}-$ основное состояние цепи. Введем $2 K$-мерный вектор $\mathbf{w}_{n}=\left\{\mathbf{u}_{n, k}-\mathbf{u}_{n, k}^{0}\right\}_{k=1}^{K}$, описывающий смещения атомов $n$-ой поперечной ячейки из своего положения равновесия. Тогда гамильтониан системы цепей (1) может быть записан в виде

$H=\sum_{n}\left[\frac{1}{2} M\left(\dot{\mathbf{w}}_{n}, \dot{\mathbf{w}}_{n}\right)+P\left(\mathbf{w}_{n-N_{0}}, \ldots, \mathbf{w}_{n}, \ldots, \mathbf{w}_{n+N_{0}}\right)\right]$.

Гамильтониану (6) соответствует система уравнений движения

$$
\begin{aligned}
& -M \ddot{\mathbf{w}}_{n}=P_{1}\left(\mathbf{w}_{n}, \mathbf{w}_{n+1}, \ldots, \mathbf{w}_{n+2 N_{0}}\right) \\
& \quad+P_{2}\left(\mathbf{w}_{n-1}, \mathbf{w}_{n}, \ldots, \mathbf{w}_{n+2 N_{0}-1}\right)+\ldots \\
& \quad+P_{2 N_{0}+1}\left(\mathbf{w}_{n-2 N_{0}}, \mathbf{w}_{n-2 N_{0}+1}, \ldots, \mathbf{w}_{n}\right), n=1,2, \ldots, N
\end{aligned}
$$

где функция

$$
\begin{aligned}
& P_{i}\left(\mathbf{w}_{1}, \ldots, \mathbf{w}_{i}, \ldots, \mathbf{w}_{2 N_{0}+1}\right) \\
& =\frac{\partial}{\partial \mathbf{w}_{i}} P\left(\mathbf{w}_{1}, \ldots, \mathbf{w}_{i}, \ldots, \mathbf{w}_{2 N_{0}+1}\right), i=1,2, \ldots, 2 N_{0}+1 .
\end{aligned}
$$

Для малых смещений в линейном приближении система уравнений (7) принимает форму

$$
\begin{aligned}
-M \ddot{\mathbf{w}}_{n}= & \mathbf{B}_{1} \mathbf{w}_{n}+\mathbf{B}_{2} \mathbf{w}_{n+1}+\mathbf{B}_{2}^{*} \mathbf{w}_{n-1}+\cdots+\mathbf{B}_{2 N_{0}+1} \mathbf{w}_{n+2 N_{0}} \\
& +\mathbf{B}_{2 N_{0}+1}^{*} \mathbf{w}_{n-2 N_{0}},
\end{aligned}
$$

где матрицы размерности $2 K \times 2 K$

$$
\begin{aligned}
& \mathbf{B}_{1}=P_{1,1}+P_{2,2}+\cdots+P_{2 N_{0}+1,2 N_{0}+1}, \\
& \mathbf{B}_{2}=P_{1,2}+P_{2,3}+\cdots+P_{2 N_{0}, 2 N_{0}+1}, \\
& \cdots \\
& \mathbf{B}_{2 N_{0}}=P_{1,2 N_{0}}+P_{2,2 N_{0}+1} \\
& \mathbf{B}_{2 N_{0}+1}=P_{1,2 N_{0}+1}
\end{aligned}
$$

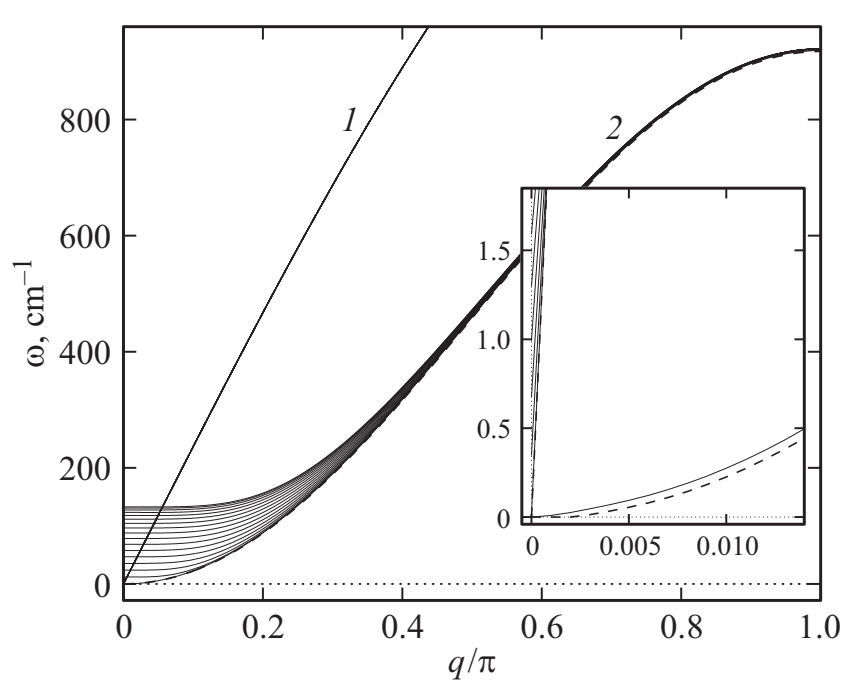

Рис. 3. Вид дисперсионных кривых листа графена из $K=1$ и $K=17$ слоев: кривые 1 описывают продольные колебания, кривые 2 - поперечные (изгибные) колебания слоев. Пунктирные кривые соответствуют колебаниям однослойного листа.

а матрицы частных производных

$$
P_{i, j}=\frac{\partial P}{\partial \mathbf{w}_{i} \partial \mathbf{w}_{j}}(\mathbf{0}, \mathbf{0}, \ldots, \mathbf{0}), \quad i, j=1,2, \ldots, 2 N_{0}+1 .
$$

Решение системы линейных уравнений (8) будем искать в форме бегущей волны

$$
\mathbf{w}_{n}(t)=A \mathbf{e} \exp (i q n-i \omega t),
$$

где $A$ - амплитуда волны (моды), е - нормализованный безразмерный вектор $[(\mathbf{e}, \mathbf{e})=1], \omega-$ частота, а $q \in[0, \pi]$ - безразмерное волновое число волны (фонона). Подставив выражение (9) в линейную систему (8), мы получим задачу на собственные значения

$$
\begin{aligned}
\omega^{2} M \mathbf{e} & =\left[\mathbf{B}_{1}+\mathbf{B}_{2} e^{i q}+\mathbf{B}_{2}^{*} e^{-i q}+\mathbf{B}_{2} e^{2 i q}\right. \\
& \left.+\mathbf{B}_{2}^{*} e^{-2 i q}+\cdots+\mathbf{B}_{2 N_{0}+1} e^{2 N_{0} i q}+\mathbf{B}_{2 N_{0}+1}^{*} e^{-2 N_{0} i q}\right] \mathbf{e} .
\end{aligned}
$$

Таким образом, для нахождения дисперсионных кривых системы параллельных цепей необходимо при каждом значении безразмерного волнового числа $q \in[0, \pi]$ найти все собственные значения эрмитовой матрицы $(10)$ размерности $2 K \times 2 K$. В результате мы получим дисперсионную кривую из $2 K$ ветвей $\left\{\omega_{j}(q)\right\}_{j=1}^{2 K}$. Одна половина ветвей будет соответствовать продольным, другая - поперечным (изгибным) колебаниям цепей (в первом случае атомы во время колебаний смещаются вдоль, во втором - поперек цепей).

Вид дисперсионных кривых для листа графена из $K=1$ и $K=17$ слоев представлен на рис. 3. Синхронные изгибные колебания всех слоев описывает самая низшая ветвь дисперсионной кривой поперечных колебаний $\omega_{1}(q)$. Как видно из рис.4, увеличение числа 
Таблица 1. Зависимость частоты изгибных колебаний однослойной ленты $\omega_{1,1}$ и отношения частоты синхронных изгибных колебаний $K$-слойной к частоте однослойной наноленты $c_{K}=\omega_{1, K} / \omega_{1,1}$ от значения безразмерного волнового числа $q / \pi$

\begin{tabular}{c|cccccc}
\hline$q / \pi$ & $\omega_{1,1}\left(\mathrm{~cm}^{-1}\right)$ & $c_{2}$ & $c_{3}$ & $c_{5}$ & $c_{9}$ & $c_{17}$ \\
\hline 0.003 & 0.014 & 2.357 & 2.714 & 2.929 & 3.000 & 3.357 \\
0.004 & 0.033 & 1.545 & 1.727 & 1.879 & 1.939 & 2.091 \\
0.005 & 0.055 & 1.327 & 1.455 & 1.564 & 1.618 & 1.709 \\
0.007 & 0.110 & 1.173 & 1.245 & 1.309 & 1.355 & 1.400 \\
0.010 & 0.226 & 1.088 & 1.128 & 1.164 & 1.195 & 1.217 \\
0.014 & 0.443 & 1.047 & 1.070 & 1.093 & 1.108 & 1.120 \\
0.020 & 0.905 & 1.024 & 1.036 & 1.049 & 1.059 & 1.065 \\
0.030 & 2.036 & 1.012 & 1.019 & 1.025 & 1.030 & 1.034
\end{tabular}

слоев $K$ приводит к слабому сдвигу этой дисперсионной кривой вверх. Величина этого сдвига $\delta \omega \approx 0.02(K=2)$, $0.03(K=3), 0.037(K=5), 0.044(K=9)$ и $0.05 \mathrm{~cm}^{-1}$ $(K=17)$. Этот сдвиг приводит к существенным изменениям частоты колебания только для длинноволновых фононов с волновым числом $q / \pi<0.01-$ см. табл. 1 .

Изменение частоты синхронного изгибного колебания $\omega_{1, K}(q)$ с увеличением числа слоев наноленты $K$ удобно описать через ее отношение к соответствующей частоте однослойной наноленты $c_{K}=\omega_{1, K} / \omega_{1,1}$. Частота изгибных колебаний однослойной наноленты $\omega_{1,1}(q) \sim q^{2}$ при $q \rightarrow \infty$, т.е. частота изгибной волны обратно пропорциональна квадрату ее длины $L=2 a \pi / q$. Из рис. 4 видно, что частота изгибной волны $\omega_{1, K}$ многослойной наноленты $(K>1)$ при $q \rightarrow 0$ убывает медленнее частоты однослойной наноленты, поэтому отношение частот $c_{K}(q)$ при малых значениях волнового числа $q$ может становиться значительно больше единицы - см. табл. 1. Так при $q=0.003 \pi$ (при длине волны изгиба $L=666.6 a=81.9 \mathrm{~nm}$ ) частота волны изгиба вследствие

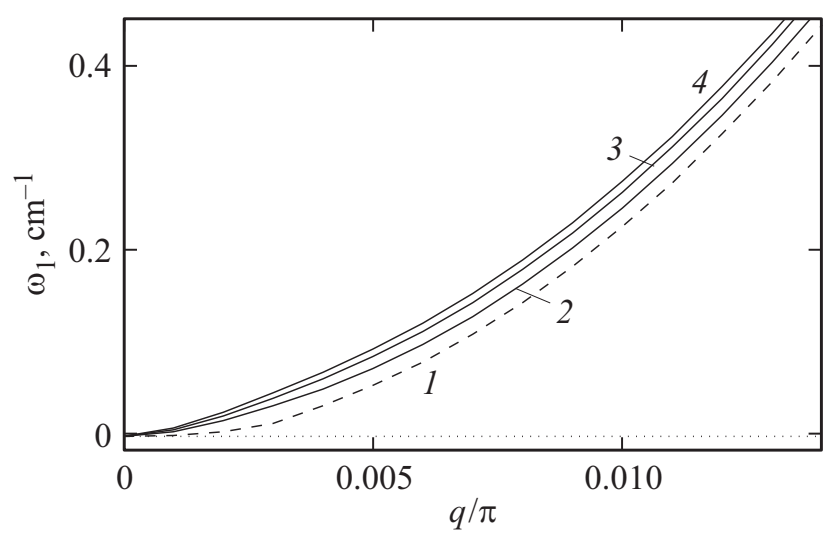

Рис. 4. Вид дисперсионных кривых синхронных изгибных колебаний $K$-слойного листа графена при $K=1,2,5,17$ (кривые $1,2,3,4)$. Показана зависимость от волнового числа $q$ наименьшей дисперсионной кривой $\omega_{1}$. взаимодействия слоев у девятислойной наноленты возрастает в три раза, соответственно жесткость изгиба наноленты при длине $L / 2=40.9 \mathrm{~nm}$ должна увеличиться в девять раз. Таким образом, можно заключить, что взаимодействие слоев приводит к существенному повышению изгибной жесткости (частоты основного изгибного колебания) только для нанолент большой длины. Для проверки этого проанализируем изгибные колебания изолированной конечной многослойной наноленты.

\section{4. Изгибные колебания конечной многослойной наноленты}

Рассмотрим $K$-слойную наноленту длины $L=(N-1) a$. В используемой модели такой наноленте соответствует система из $K$ параллельных цепей из $N$ звеньев каждая (см. рис. 1). Гамильтониан системы цепей имеет вид (1). Вначале найдем основное стационарное состояние системы. Для этого численно решим задачу на минимум потенциальной энергии системы

$$
E \rightarrow \min :\left\{x_{n, k}, z_{n, k}\right\}_{n=1, k=1}^{N, K} .
$$

Пусть $\left\{x_{n, k}^{0}, z_{n, k}^{0}\right\}_{n=1, k=1}^{N, K}-$ решение этой задачи.

Найдем собственные колебания $K$-слойной конечной наноленты. Гамильтониану (10) соответствует система уравнений движения

$$
\begin{gathered}
M \ddot{x}_{n, k}=-\frac{\partial H}{\partial x_{n, k}}, \quad M \ddot{z}_{n, k}=-\frac{\partial H}{\partial z_{n, k}}, \\
n=1, \ldots, N, \quad k=1, \ldots, K
\end{gathered}
$$

Рассмотрим малые смещения из основного состояния системы. Для этого сделаем замену $x_{n, k}=x_{n, k}^{0}+u_{n, k}$, $z_{n, k}=z_{n, k}^{0}+v_{n, k}$. Для малых смещений из положения равновесия $\left|u_{n, k}\right| \ll a,\left|v_{n, k}\right| \ll a$ система уравнений движения (12) может быть записана как система линейных уравнений размерности $2 \mathrm{NK}$

$$
-M \ddot{\mathbf{x}}=\mathbf{C x},
$$

где вектор $\mathbf{x}=\left\{u_{n, k}, v_{n, k}\right\}_{n=1, k=1}^{N, \quad K}, \mathbf{C}-$ положительно определенная симметричная матрица вторых производных потенциальной энергии системы цепей в основном состоянии. Собственному колебанию наноленты соответствует решение системы линейных уравнений (13)

$$
\mathbf{x}_{l}(t)=A \mathbf{e}_{l} \exp \left(-i \omega_{l} t\right)
$$

где $\omega_{l}^{2}$ и $\mathbf{e}_{l}-l$-тое собственное значение и соответствующий собственный вектор матрицы $\mathbf{C} / M$. Первые три собственных значения всегда равны нулю, этим значениям соответствует движение системы параллельных цепей по плоскости $x z$ как твердого тела.

Рассмотрим собственное колебание с наименьшей ненулевой частотой, при котором все слои наноленты 
Таблица 2. Зависимость частоты изгибных колебаний однослойной ленты $\omega_{b, 1}$ и отношения частоты синхронных изгибных колебаний $K$-слойной к частоте однослойной наноленты $c_{K}=\omega_{b, K} / \omega_{b, 1}$ от числа звеньев цепи $N$ (длина наноленты $L=(N-1) a)$

\begin{tabular}{r|cccccc}
\hline \multicolumn{1}{c|}{$N$} & $\omega_{b, 1}\left(\mathrm{~cm}^{-1}\right)$ & $c_{2}$ & $c_{3}$ & $c_{5}$ & $c_{9}$ & $c_{17}$ \\
\hline 25 & 8.217 & 1.028 & 1.042 & 1.055 & 1.066 & 1.078 \\
50 & 2.053 & 1.044 & 1.065 & 1.084 & 1.094 & 1.096 \\
100 & 0.513 & 1.082 & 1.127 & 1.191 & 1.241 & 1.263 \\
200 & 0.128 & 1.328 & 1.445 & 1.586 & 1.695 & 1.711 \\
300 & 0.057 & 1.614 & 1.789 & 2.105 & 2.105 &
\end{tabular}

синхронно изгибаются (см. рис. 5). Зависимость частоты этого колебания $\omega_{b, K}$ от длины (числа звеньев цепи $N$ ) и толщины (числа параллельных цепей $K$ ) изолированной наноленты представлена в табл. 2. Как видно из таблицы, частота изгибных колебаний однослойной наноленты с увеличением ее длины уменьшается как обратный квадрат длины (увеличение в два раза длины приводит к уменьшению в четыре раза частоты). Частота колебаний начинает существенно зависеть от толщины наноленты только при $N>100$ (при длине $L>12 \mathrm{~nm}$ ). Чем больше длина наноленты, тем сильнее межцепное взаимодействие слоев приводит к увеличению частоты изгибных колебаний.

Анализ колебаний показывает, что изгибное колебание изолированной многослойной наноленты всегда сопровождается скольжением ее слоев. Особенно сильно скольжение происходит у ее краев. Из формы собственного колебания (см. рис. 5) видно, что при изгибе поперечные слои атомов остаются линейными (поперечные сечения наноленты остаются плоскими), но не остаются ортогональными линии изгиба. Поэтому для описания
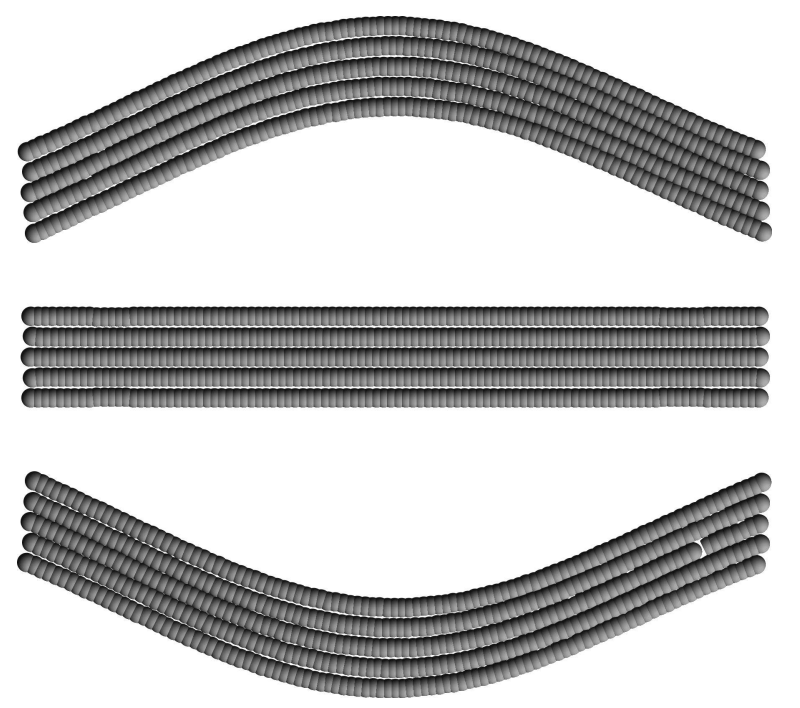

Рис. 5. Вид основного изгибного колебания пятислойной наноленты графена (число узлов каждого слоя $N=100$, число слоев $K=5$, частота колебания $\omega_{b}=0.611 \mathrm{~cm}^{-1}$ ). изгиба многослойной наноленты нельзя использовать классическую теорию изгиба балок Эйлера-Бернулли, а нужно использовать теорию изгиба балок Тимошенко [15].

\section{5. Колебания многослойной консоли}

Возьмем $K$-слойную конечную наноленту, расположенную вдоль оси $x$ параллельно плоскости $x y$, и зафиксируем положение ее левых краевых звеньев с номерами $n=1,2, k=1, \ldots, K$ (см. рис. 1$)$. Тем самым мы получим многослойную консоль длины $L=(N-1) a$. Зависимость частоты основного изгибного колебания консоли $\omega_{g, K}$ от длины консоли и числа ее слоев представлена в табл. 3. Как видно из таблицы, для однослойной консоли частота $\omega_{b, 1}$ с увеличением длины убывает как ее обратный квадрат (увеличение длины в два раза приводит к уменьшению частоты в четыре раза). Взаимодействие слоев консоли приводит к заметному увеличению частоты основного изгибного колебаний только при $N>100(L>12 \mathrm{~nm})$. Колебания сопровождаются скольжением слоев, особенно сильным у свободного края консоли. В результате этого при изгибе поперечные слои атомов консоли не остаются ортогогальными линии изгиба (см. рис. 6).

Жесткость многослойной консоли на изгиб можно также оценить из зависимости энергии стационарного изгиба консоли $E$ от величины вертикального сдвига ее правого углового атома $h-$ см. рис. 6. Зависимость квадратного корня нормированной энергии стационар-

Таблица 3. Зависимость частоты основного изгибного колебания однослойной консоли $\omega_{b, 1}$ и отношения частоты синхронных изгибных колебаний $K$-слойной к частоте колебаний однослойной консоли $c_{K}=\omega_{b, K} / \omega_{b, 1}$ от числа звеньев цепи $N$ $(K$-слойная нанолента длины $L=(N-1) a$ с закрепленным левым краем).

\begin{tabular}{r|cccccc}
\hline$N-2$ & $\omega_{b, 1}\left(\mathrm{~cm}^{-1}\right)$ & $c_{2}$ & $c_{3}$ & $c_{5}$ & $c_{9}$ & $c_{17}$ \\
\hline 12 & 4.786 & 1.028 & 1.041 & 1.052 & 1.057 & 1.059 \\
24 & 1.291 & 1.040 & 1.059 & 1.077 & 1.088 & 1.087 \\
48 & 0.336 & 1.033 & 1.048 & 1.060 & 1.074 & 1.080 \\
96 & 0.087 & 1.034 & 1.092 & 1.115 & 1.253 & 1.299 \\
144 & 0.039 & 1.282 & 1.385 & 1.769 & 1.872 &
\end{tabular}

Таблица 4. Зависимость коэффициента изгибной жесткости $\kappa$ от длины (от числа звеньев $N$ ) и толщины (числа слоев $K$ ) консоли. Размерность коэффициента $[\kappa]=\mathrm{eV} \AA$

\begin{tabular}{r|crrrr}
\hline \multicolumn{1}{c|}{$N$} & $K=1$ & $K=2$ & $K=3$ & $K=5$ & $K=9$ \\
\hline 25 & 6.87 & 7.55 & 7.86 & 8.09 & 8.04 \\
50 & 6.65 & 7.35 & 7.66 & 7.95 & 8.15 \\
100 & 6.55 & 7.67 & 8.65 & 11.10 & 13.18 \\
200 & 7.00 & 21.41 & 25.14 & 36.46 & 41.97
\end{tabular}




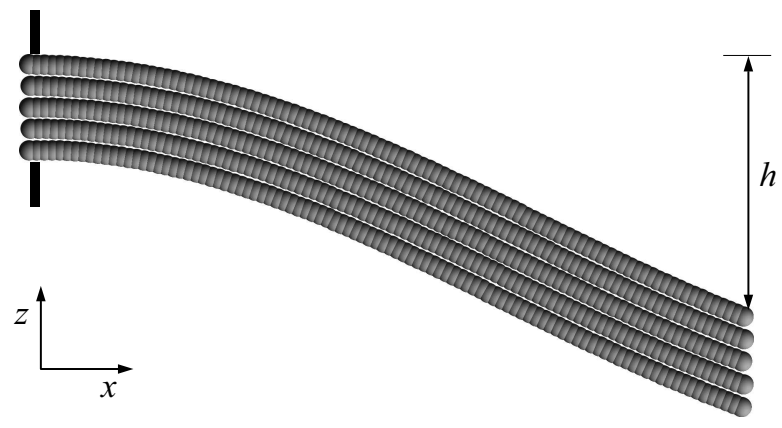

Рис. 6. Стационарный изгиб консоли из пятислойной наноленты длины $L=99 a=12.16 \mathrm{~nm}$ (число звеньев цепи $N=100$ ) при вертикальном смещении ее правого края на $h=4 \mathrm{~nm}$ $(h / L=0.329)$.

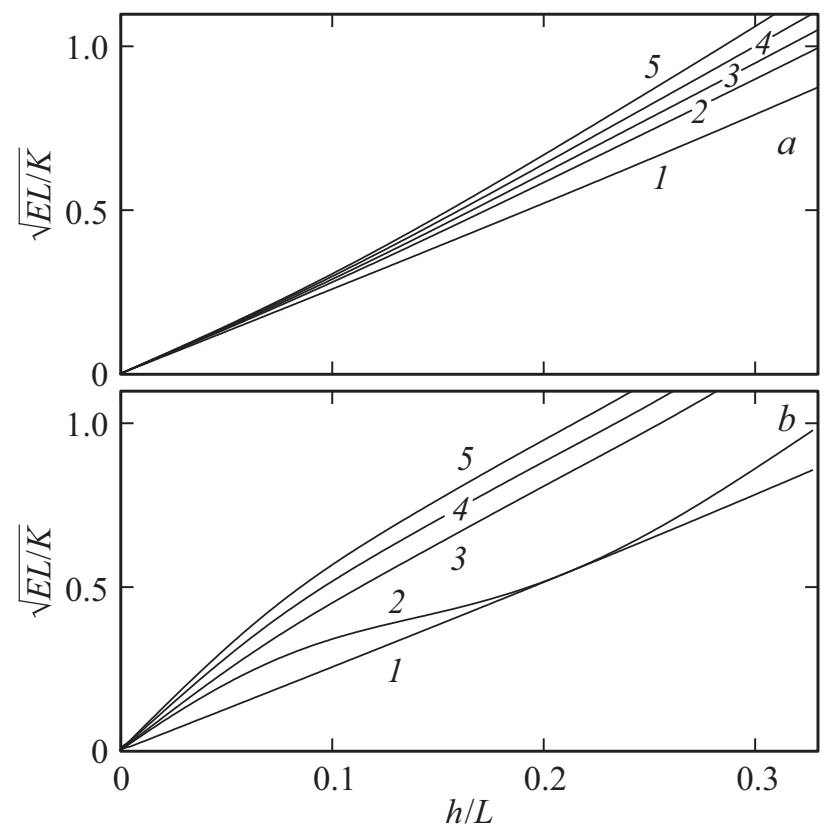

Рис. 7. Зависимость квадратного корня нормированной энергии стационарного изгиба многослойной консоли $\sqrt{E L / K}$ от безразмерной величины вертикального сдвига ее правого края $h / L$ при числе звеньев в слое $(a) N=50$ и $(b) N=200$. Кривые $1,2,3,4$ и 5 дают зависимость для наноленты из $K=1,2,3,5$ и 9 слоев.

ного изгиба консоли $\sqrt{E L / K}$ от безразмерного значения вертикального сдвига правого края $h / L$ представлена на рис. 7.

Решение задачи на минимум энергии изгибного состояния консоли показало, что при $h \rightarrow 0$ энергия изгиба всегда уменьшается как $h^{2}$, поэтому удобно ввести параметр пропорциональности

$$
\kappa=\lim _{h \rightarrow 0} \frac{E L / K}{(h / L)^{2}}=\frac{L^{3}}{K} \lim _{h \rightarrow 0} E / h^{2},
$$

характеризующий изгибную жесткость консоли, нормированную на ее длину и толщину. Значения этого коэффициента изгибной жесткости наноленты при разных значениях $N$ и $K$ представлены в табл. 4. Как видно из таблицы, взаимодействие слоев консоли приводит к существенному увеличению ее жесткости только при длинах $L>12 \mathrm{~nm}(N>100)$. Так при длине $L=24.4 \mathrm{~nm}$ $(N=200)$ взаимодействие слоев приводит к увеличению изгибной жесткости в 3 раза для двухслойной, и в 6 раз для девятислойной нанолетны.

\section{6. Заключение}

Проведенный в работе анализ изгибных колебаний бесконечных многослойных нанолент и прямое численное моделирование колебаний и изгибов конечных нанолент позволяют заключить, что слабое невалентное взаимодействие слоев приводит к заметному повышению нормированной на число слоев изгибной жесткости только для нанолент длины $L>12 \mathrm{~nm}$. Чем больше длина, тем сильнее происходит это повышение. Так при длине $L=24 \mathrm{~nm}$ взаимодействие слоев приводит к увеличению изгибной жесткости в три раза для двухслойной и в шесть раз для девятислойной наноленты. Поэтому использование многослойных нанолент может позволить существенно увеличить устойчивость к изгибу протяженных наноконструкций.

\section{Список литературы}

[1] K.S. Novoselov, A.K. Geim, S.V. Morozov, D. Jiang, Y. Zhang, S.V. Dubonos, I.V. Grigorieva, A.A. Firsov. Science 306, 666 (2004).

[2] A.K. Geim, K.S. Novoselov. Nature. Mater. 6, 183 (2007).

[3] C. Soldano, A. Mahmood, E. Dujardin. Carbon 48, 2127 (2010).

[4] J.A. Baimova, B. Liu, S.V. Dmitriev, K. Zhou. Phys. Status Solidi R 8, 336 (2014).

[5] J.A. Baimova, B. Liu, S.V. Dmitriev, N. Srikanth, K. Zhou. Phys. Chem. Chem. Phys. 16, 19505 (2014).

[6] J.A. Baimova, E.A. Korznikova, S.V. Dmitriev, B. Liu, K. Zhou. Rev. Adv. Mater. Sci. 39, 69 (2014).

[7] A.V. Orlov, I.A. Ovid'ko. Rev. Adv. Mater. Sci. 40, 249 (2015).

[8] O.K. Kwon, J.H. Lee, K.-S. Kim, J.W. Kang. Physica E 47, 6-11 (2013).

[9] S.I. Ahn, J.R. Jung, S.Y. Choi, M.H. Son, Y.J. Hong, J.-C. Park. Sci. Rep. 7, 12604 (2017).

[10] S.P. Milovanovic, M.Z. Tadic, F.M. Peeters. Graphene membrane as a pressure gauge. Appl. Phys. Lett. 111, 043101 (2017).

[11] C. Berger, R. Phillips, A. Centeno, A. Zurutuza, A. Vijayaraghavan, Nanoscale 9, 17439-17449 (2017).

[12] A.V. Savin, E.A. Korznikova, S.V. Dmitriev. Phys. Rev. B 92, 035412 (2015).

[13] А.В. Савин, Е.А. Корзникова, С.В. Дмитриев. ФТТ 57, 2278 (2015).

[14] А.В. Савин, Е.А. Корзникова, И.П. Лобзенко, Ю.А. Баимова, С.В. Дмитриев. ФТТ 58, 1236 (2016).

[15] R. Nazemnezhad. Comp. Struct. 133, 522-528 (2015).

Редактор Т.Н. Василевская 